\title{
LA FUNCIÓN DE LA LECTURA DE TEXTOS EN INGLÉS EN EL DESARROLLO COGNITIVO: EL USO DE PREGUNTAS ORIENTADORAS DURANTE EL PROCESO DE COMPRENSIÓN Y RETENCIÓN DE LA INFORMACIÓN
}

\author{
Luisa María González Rodríguez y Ana Alonso Alonso. Universidad de \\ Salamanca
}

\begin{abstract}
La finalidad de este artículo es destacar las posibilidades que ofrece la lectura de textos en inglés para adquirir conocimientos que no sean estrictamente lingüísticos. Hay numerosos estudios que consideran que el uso de estrategias cognitivas y metacognitivas favorece el proceso de aprendizaje. Es, pues, nuestra intención explicar cómo los profesores podemos enseñar y practicar estas estrategias mediante el uso de preguntas orientadoras que ayuden al alumno a mejorar su capacidad lectora. Por último, incluiremos las consecuencias pedagógicas que se derivan del uso de esta metodología.
\end{abstract}

\section{INTRODUCCIÓN}

Gran parte de los conocimientos que adquirimos a lo largo de nuestra vida provienen de las lecturas que realizamos en nuestra lengua materna. Sin embargo, al estudiar otro idioma, la finalidad de la lectura parece ser únicamente el aprendizaje de las estructuras gramaticales y el vocabulario de dicho idioma. Los profesores utilizamos los textos de una manera artificial y los explotamos didácticamente utilizando preguntas que, en ocasiones, no ayudan ni a entender mejor el texto ni a aprender nada de él. Por este motivo, los alumnos suelen tener la sensación de que se lee un texto, para contestar unas preguntas que no les interesan y que sólo sirven para distraerlos del proceso de comprensión. La lectura se convierte así en algo monótono, desmotivador, y, lo que es peor, se percibe como una actividad carente de sentido y utilidad.

Por este motivo, proponemos como alternativa la utilización de un método cognitivo basado en la adquisición de conocimientos a través de la lectura de textos en inglés (Sorani y Tamponi, 1992; Badrawi,1992). Los alumnos estarán más motivados pues al leer complementarán los conocimientos que han adquirido en otras asignaturas. Además, al 
integrar el aprendizaje de estrategias de comprensión con la adquisición de nuevos conocimientos, la actividad de la lectura adquirirá una dimensión más profunda y compleja. Esta complejidad, exige al alumno/lector una implicación activa en el proceso. Se hace pues necesario el uso de los conocimientos previos del lector y de una serie de estrategias cognitivas y metacognitivas que faciliten la comprensión y retención de la información del texto. Estas estrategias permitirán al alumno darse cuenta de la estructura del texto, distinguir las ideas principales, deducir las ideas implícitas, ignorar los detalles y contribuirán a ayudarle a procesar activamente los nuevos conocimientos para integrarlos en su sistema cognitivo.

En ocasiones los profesores no enseñamos estas estrategias porque pensamos que es algo que se desarrolla progresivamente sin necesidad de ayuda. Sin embargo, hay numerosos estudios de psicología cognitiva (Garner, 1988; Stevens, 1988; Yuill y Joscelyne, 1988) que demuestran que la enseñanza y práctica de determinadas estrategias de comprensión textual para controlar y favorecer el progreso cognitivo del lector han dado buenos resultados, especialmente en casos en que el lector no las usa de manera espontánea (como es el caso de la lectura en otro idioma). Por consiguiente, consideramos necesario modificar las actividades de comprensión para fomentar el uso y la práctica de dichas estrategias. En este trabajo, centraremos la atención en la función orientadora de las preguntas de comprensión en la difícil tarea de procesar y retener la información textual de una manera eficaz. Analizaremos las estrategias que necesitan nuestros alumnos para convertirse en lectores autónomos y el modo de usar las preguntas de comprensión para conseguir los objetivos propuestos.

\section{ESTRATEGIAS COGNITIVAS Y METACOGNITIVAS}

Las estrategias de comprensión pueden definirse como actividades controladas y planeadas por el lector para comprender mejor un texto, de forma que permitan controlar el proceso de comprensión y retención de la información (Oxford, 1990; O’Malley y Chamot, 1990). Distinguiremos entre estrategias cognitivas, cuya finalidad es el progreso cognitivo (el aprendizaje), y estrategias metacognitivas, cuya función es supervisar y controlar el proceso de aprendizaje.

En este trabajo no mencionaremos las estrategias lingüísticas (ej: deducir el significado de una palabra por el contexto, interpretar la cohesión léxica...) básicas para una comprensión superficial del texto por creer que éstas deben ser aprendidas en las primeras fases del aprendizaje y porque, en nuestra opinión, reciben suficiente atención en la mayoría de los libros de texto. Además, centrarse excesivamente en el vocabulario puede consolidar el uso de estrategias de lectura negativas (Block, 1992:338). Describiremos a continuación algunas de las estrategias fundamentales para acceder a los textos a un nivel más profundo y significativo:

\subsection{Predicción de la información}

Esta estrategia es importante porque activa los conocimientos previos del lector/ alumno y permite la interacción entre la información ya conocida y la nueva. La utilización de esta estrategia facilita la comprensión porque puede ayudar a anticipar la estructura en virtud de la cual vamos a interpretar el texto. Como consecuencia se lee más rápido para confirmar o rechazar las expectativas.

Odisea, $n^{\circ} 2,2002$ 


\subsection{Resumen de las ideas del texto}

Ésta es una estrategia compleja que los lectores expertos realizan mentalmente durante el proceso de la lectura. Resumir inmediatamente lo que se lee ayuda a recordar las ideas y comprender mejor el resto del texto. Para Garner (1988) resulta muy provechoso resumir las ideas de cada párrafo en los textos cortos y antes de cada nuevo epígrafe si el texto es largo. Esta estrategia cognitiva obliga a utilizar otras más simples:

a) distinguir entre las ideas principales y secundarias

b) ignorar los detalles

c) parafrasear la información del texto

d) elaborar, reorganizar y sintetizar la información.

Además, resumir las ideas durante la lectura resulta muy útil como estrategia metacognitiva para controlar si se está comprendiendo bien el texto. Así pues, en caso de no poder resumir las ideas, nos plantearíamos que estamos ante un problema de comprensión y necesitaríamos recurrir a otras estrategias para solucionarlo (ej: releer el texto...). En palabras de Garner (1988:22) "this situation can be characterized as cognitive failure, metacognitive success". El hecho de que el lector se dé cuenta de que no ha entendido bien es prueba de que funcionan bien las estrategias de comprobación y control.

\subsection{Relectura de partes del texto}

Es una estrategia que consiste en volver a leer intencionadamente partes concretas del texto. Por supuesto, releer el texto entero sería utilizar la estrategia de forma inapropiada. Se utiliza cuando necesitamos recordar ideas, solucionar problemas de inconsistencia entre las ideas o buscar información que anteriormente nos pasó desapercibida. Dentro de ésta podríamos incluir las siguientes estrategias:

a) darse cuenta de que no recordamos algo y que la información está en el texto.

b) escanear el texto rápidamente para localizar el párrafo o frase que contiene la información que buscamos.

c) combinar ideas para deducir ideas implícitas.

\subsection{Deducción de ideas implícitas}

Al igual que las anteriores es una estrategia compleja que obliga a utilizar otras más simples:

a) utilización del contexto para formular suposiciones y sacar conclusiones.

b) reorganizar y combinar las ideas del texto para establecer conexiones que no se expresen explícitamente.

c) utilizar los conocimientos previos

d) aplicar las ideas del texto a nuevas situaciones.

La utilización de esta estrategia implica que hemos extraído el significado del texto y estamos interesados en las ideas subyacentes como: la intención del autor, el tono irónico, etc...

\subsection{Organización del discurso}

Hay numerosos estudios que avalan la afirmación de que saber cómo está estructurado un texto facilita la comprensión y aumenta la capacidad mnemónica (Voss y Silfies,1996). Su uso durante el proceso de lectura conlleva: 
a) saber interpretar los marcadores del discurso que nos indican el orden cronológico de los acontecimientos;

b) saber interpretar las expresiones que nos indican si el autor está enfatizando una idea, poniendo ejemplos para ilustrar algo, expresando de otra manera algo que ya se había mencionado, etc.;

c) distinguir las ideas principales y ordenarlas jerárquicamente;

d) reorganizar la información de manera selectiva;

e) distinguir la relación entre las diferentes partes del texto;

f) distinguir los hechos de las opiniones y lo general de lo particular;

g) valorar el punto de vista y la intención del autor.

\subsection{Supervisión o control de la comprensión}

Todas las estrategias anteriores necesitan complementarse con la capacidad de supervisar y evaluar el proceso de comprensión. Sólo así se podrán detectar los problemas y solucionarlos de la manera adecuada (Casanave,1988). Para dominar esta estrategia metacognitiva es necesario utilizar habitualmente mecanismos de control y solución de problemas. Normalmente se realiza de manera automática y sólo cuando se detectan los problemas de comprensión (inconsistencia entre ideas, conceptos difíciles de manejar o asimilar, ambigüedad de ideas, etc...) el alumno necesitará:
a) releer partes del texto
b) continuar leyendo
c) parafrasear la información
d) hacer preguntas al profesor, a otros alumnos o a sí mismo
e) recurrir al diccionario o a otras fuentes de información o
f) utilizar sus conocimientos previos.

Las estrategias metacognitivas permiten a los alumnos reflexionar sobre el modo en que aprenden y les conciencian de que los conocimientos se desarrollan en función de las necesidades. Todo lo cual les permitirá analizar previamente la finalidad de la lectura, plantearse unos objetivos y comprobar si se van alcanzando durante el proceso de comprensión del texto. Además, se acostumbrarán a considerar el texto desde una perspectiva comunicativa, es decir, centrarán su atención en descifrar la información procesando automáticamente los elementos gramaticales.

El uso continuado y sistemático de todas estas estrategias contribuirá a formar lectores más expertos y autónomos, es decir, lectores que disfruten y aprendan durante el proceso de lectura.

\section{LAS PREGUNTAS ORIENTADORAS}

En los últimos años, las actividades de comprensión propuestas por los libros de texto de inglés han experimentado importantes cambios en cuanto a su flexibilidad y variedad. Sin embargo, pensamos que todavía siguen centrando su atención en el aprendizaje de la gramática y del vocabulario menospreciando la posibilidad de fomentar la adquisición de nuevos conocimientos a través de la lectura. Este excesivo énfasis sobre los aspectos formales contribuye a dar una visión fragmentaria del texto, e impide la comprensión global del texto como discurso.

Por otra parte, la mayoría de las preguntas están pensadas para evaluar la competencia del alumno y no para desarrollar su capacidad de comprensión (Nuttall, 1982). Las 
preguntas de comprensión se han convertido, así, en sinónimo de preguntas de evaluación. Por ello, hemos decidido utilizar el término de preguntas orientadoras para referirnos a aquellas preguntas que contribuyen a desarrollar y a aplicar estrategias cognitivas y metacognitivas de comprensión textual. La función de estas preguntas debe ser facilitar la lectura $\mathrm{y}$, en ningún caso, entorpecer ni distraer al alumno de lo que es nuestro verdadero objetivo, a saber, la comprensión del texto en toda su dimensión. Estas preguntas dirigen la atención del alumno hacia los aspectos esenciales del texto, le conciencian de que hay partes que no entiende y le enseñan a aplicar estrategias para salvar las dificultades que surjan durante la lectura. Es importante que el profesor las utilice sistemáticamente para ayudar a los alumnos a entender la estructura del texto, a deducir las ideas implícitas, a relacionar la información de las diferentes secciones y, especialmente, para que desarrollen estrategias metacognitivas que les permitan reflexionar sobre el proceso de comprensión de un texto. Existen muchos tipos de preguntas y no hay unos mejores que otros, la cuestión es usarlas adecuadamente para conseguir los objetivos propuestos. Algunas preguntas ayudarán a los alumnos a discriminar las ideas principales o a parafrasear la información, otras a relacionar ideas, pero todas deben integrarse y complementarse para activar y mejorar la comprensión y retención.

La tarea del profesor será la de seleccionar preguntas relevantes e interesantes que obliguen al alumno a involucrarse activamente en el proceso de lectura y que generen discusiones para aclarar el significado del texto. Como consecuencia, los alumnos entenderán y recordarán mejor lo que leen. Además, si se crea un clima adecuado en clase y el profesor concede más importancia a las respuestas bien razonadas que a las respuestas correctas, los alumnos participarán más, perderán el miedo a equivocarse y serán conscientes de sus progresos cognitivos. Progresivamente, los alumnos podrán observar los efectos beneficiosos de estas preguntas que les sirven de guía para entender el texto y serán capaces de utilizar este método cuando lean. Razonar las respuestas en clase y reflexionar sobre el proceso de comprensión les permitirá depender cada vez menos de la ayuda del profesor y convertirse en lectores expertos. Asimismo, el método propuesto les ayudará a aprender el idioma a la vez que adquieren otro tipo de conocimientos. Y a disfrutar así de su lectura.

A continuación presentamos una clasificación de tipos de preguntas orientadoras según el grado de dificultad y el nivel de comprensión textual que exigen al alumno. Para ello hemos adaptado la taxonomía de Nutall (1982):

\subsection{Preguntas de comprensión literal}

Son preguntas cuyas respuestas aparecen explícitamente en el texto. No requieren mucho esfuerzo mental por parte del alumno y en ocasiones se pueden responder con las mismas palabras que el texto y sin haberlo entendido. Sin embargo, el profesor puede evitar que esto suceda pidiendo a los alumnos que expliquen su respuesta con otras palabras o parafraseando él mismo las frases del texto que éstos usan al responder. Estas preguntas pueden utilizarse con la finalidad de centrar la atención del alumno sobre ideas o aspectos importantes del texto. Además les ayudan a practicar estrategias -releer partes del texto de forma selectiva o ignorar los detalles- que son fundamentales para facilitar la comprensión y la retención de las ideas del texto. Por ejemplo, si en un texto sobre la corrosión preguntamos, "What is corrosion?" o "How can corrosion be controlled?", los alumnos se darán cuenta de que es importante saber la definición del mencionado proceso y los modos

Odisea, $\mathrm{n}^{\mathrm{o}} 2,2002$ 
de frenarlo. De esta forma, al fijarse y releer esa información, se recordarán mejor las ideas principales.

\subsection{Preguntas que requieren reorganizar o interpretar las ideas del texto}

Las respuestas se encuentran también en el texto pero para contestarlas hay que considerar el texto en su conjunto y reunir información de diferentes partes del mismo. Su utilización permite a los alumnos seleccionar y reorganizar la información que aparece a lo largo del texto de una forma más coherente que facilite su recuerdo. Dentro de este epígrafe podrían incluirse preguntas como "What are the disadvantages of friction?", "What factors influence the choice of a lubricant?"

\subsection{Preguntas para deducir ideas implícitas}

Este tipo de preguntas resultan más difíciles que las anteriores pues requieren la utilización de varias estrategias de comprensión al mismo tiempo. Ayudan al alumno a establecer conexiones entre ideas, a reconocer y suplir las lagunas de información o a aplicar sus conocimientos previos sobre el tema. De esta forma, el alumno se implica activamente en el proceso de comprensión $\mathrm{y}$, como consecuencia, descifrará mejor el mensaje y recordará mejor la información textual.

\subsection{Preguntas de solución de problema}

Estas preguntas valoran la capacidad de complementar la información textual con los conocimientos previos para resolver el problema. Exigen bastante esfuerzo mental pues deben aplicar las ideas del texto a una nueva situación, pero les resultan mucho más interesantes y útiles. Por ejemplo se les puede pedir a los alumnos que lean un texto y se les pregunta, "What does this experiment show?"; o bien contestan a la pregunta: "If the beam shown in Diagram 1 were two metres longer, what effect would this have on the bending moments?", utilizando la información del texto y el diagrama que lo acompaña.

Para contestar estas preguntas los alumnos deben entender el texto a un nivel más profundo. En el primer ejemplo se les pide que saquen conclusiones que no están expresadas de forma explícita y que apliquen sus conocimientos previos. En el segundo ejercicio los alumnos necesitan entender la información verbal y completarla con la de la ilustración para resolver el problema. La realización de estas actividades es muy gratificante para los alumnos pues se dan cuenta de que su progreso en la comprensión de textos en inglés les sirve para ampliar sus conocimientos sobre otros temas y aplicarlos a nuevas situaciones.

\subsection{Preguntas de valoración del texto}

Los alumnos tienen que opinar sobre la intención del autor y sobre cómo consigue sus objetivos. También les ayuda a observar si las ideas del texto están expresadas objetiva o subjetivamente. Estas preguntas son muy importantes porque utilizan estrategias que les orientan sobre la estructura del discurso y contribuyen a desarrollar una actitud crítica frente a la lectura: ver si el autor manipula u oculta datos, si sus opiniones defienden unos determinados intereses, etc.

En determinados tipos de texto es importante saber cuál es la intención del autor y la audiencia a quien se dirige para entender bien el mensaje. Sin embargo, al ser preguntas 
difíciles de contestar es necesario hacer otras preguntas previas que orienten al alumno. A continuación proponemos un ejemplo de cómo hacerlo: Elegimos un texto sobre los peligros del amianto y les pedimos que contesten las siguientes preguntas:

1. What is the writer's aim?

2. Who is the text intended for?

Para facilitarles las respuestas podemos hacer algunas preguntas orientadoras:

a) Why does the author mention first the advantages and then the disadvantages?

b) Do you think that statistical data can be manipulated? How?

También se pueden usar preguntas de opción múltiple:

1.Was the passage intended for:
a. specialists?
b. students?
c. general readers?
d. government, industry, workers and general public?

2. Is the purpose of the excerpt:

a. to interest people in a new subject?

b. to provide new information?

c. to persuade people to adopt a particular point of view?

La realización de estos ejercicios proporciona a los alumnos la oportunidad de aprender y practicar determinadas estrategias de comprensión que podrán aplicar en sus futuras lecturas. Por ello pensamos que los profesores no sólo podemos sino que debemos enseñar a los alumnos a usar estrategias que no utilizan normalmente para que consigan leer y aprender de la forma más adecuada.

\subsection{Preguntas sobre reacciones personales}

Para contestar estas preguntas el alumno necesita haber entendido y procesado la información del texto. Se le pide que exprese sus opiniones y reacciones, pero es necesario que se base en las ideas del texto para contestar. Las respuestas deben ser razonadas para que el alumno reflexione sobre los aspectos del texto que han sugerido esas opiniones. Este tipo de preguntas resultan menos artificiales porque tienen en cuenta el factor afectivo. Los alumnos se dan cuenta de que cuando leen aprenden, disfrutan y pueden contrastar la nueva información con sus conocimientos previos. De esta forma conseguimos otro de nuestros objetivos, que integren las ideas del texto en su sistema cognitivo.

\section{CÓMO Y CUÁNDO USAR LAS PREGUNTAS ORIENTADORAS}

Las preguntas orientadoras deben utilizarse intentando emular la naturalidad con que se plantean y superar los obstáculos durante el proceso de comprensión y memorización de los contenidos. Para ello proponemos que se hagan preguntas antes, durante y después de la lectura:

\subsection{Antes de la lectura}

Antes de la lectura se pueden plantear preguntas abiertas con la finalidad de activar los conocimientos previos sobre el tema y los esquemas sobre la posible organización del texto. Es necesario que las preguntas ayuden al alumno a centrarse en los aspectos más importantes del texto. La función de realizar preguntas previas a la lectura es motivar al 
alumno, al darle una razón para leer, para que se concentre posteriormente en el proceso de lectura. Por ejemplo, se les puede pedir que lean el título o subtítulos para ver cómo está organizado un texto o que utilicen las ilustraciones y/o esquemas para predecir las ideas. También se les da una serie de ideas principales y se les hace leer rápidamente el texto para identificar los párrafos que desarrollan estas ideas. Esta pregunta además de ayudarles a ver la organización del texto les permite darse cuenta de que cada párrafo desarrolla una idea. En ocasiones podemos hacer preguntas concretas como "Why doesn't a ship sink?", antes de leer un tema sobre fuerzas en ingeniería. Al trabajar previamente sobre ejemplos concretos les ayudaremos a entender mejor las explicaciones y generalizaciones del texto. De esta forma, además orientaremos y enfocaremos su atención hacia los aspectos más importantes.

\subsection{Durante la lectura}

Las preguntas que hagamos durante la lectura no deben distraer al alumno durante el proceso de comprensión. En este momento las mejores preguntas son las de verdadero/ falso o las de opción múltiple pues son las que más se parecen a las que un lector se plantea mentalmente durante la lectura (Widdowson, 1978). La función de estas preguntas es facilitar la retención y la relación entre las ideas del texto. Por ejemplo, en un texto sobre máquinas ideales y prácticas se intercalan en la mitad tres afirmaciones para responder verdadero o falso. Esto les obliga a aclarar algunos conceptos antes de pasar a la segunda parte que es más complicada. El ejercicio es sencillo pero el interés está en el razonamiento que se haga de las respuestas. La primera afirmación pretende aclarar el significado de ventaja mecánica, un concepto que es necesario entender y recordar para comprender mejor la segunda parte. La segunda tiene como objetivo que el alumno se plantee si ha entendido bien una de las frases del texto que es un poco ambigua. En la tercera afirmación el alumno tiene que comprender tanto la información textual como el diagrama. Para responder estas preguntas el alumno debe utilizar las estrategias de releer, resumir, aclarar conceptos, relacionar ideas y en la última además tiene que controlar si ha entendido bien y es capaz de aplicar lo que ha entendido a una nueva situación.

\subsection{Después de la lectura}

Después de la lectura debemos intentar que las preguntas den una visión del texto como discurso. Las preguntas más adecuadas serán las que obliguen al alumno a relacionar ideas, a valorar el texto y expresar sus opiniones. Se les puede pedir a los alumnos que comparen varias fuentes de información sobre el mismo tema con la finalidad de:

1. Obtener información complementaria.

2. Encontrar un texto que explique mejor el tema y les resulte más asequible.

3. Analizar las similitudes y las diferencias.

4. Comprender mejor la información con la ayuda de tablas, gráficos o mapas.

Es necesario que las actividades que realicen no sólo les ayuden a procesar la información del texto sino también a integrar los nuevos conocimientos en su sistema cognitivo para ser capaces de expresar sus opiniones. Por ejemplo, en un texto sobre el proceso de desertización se pueden hacer las siguientes preguntas:

1. Explain the relationship between the growing of crops, overgrazing, malnutrition and desertification.

Odisea, $\mathrm{n}^{\mathrm{0}} 2,2002$ 
2. How would you feel if you lived in the Sahel area?

3. What could be done to stop this problem?

4. Give your opinion about how the consequences of desertification could affect developed countries.

\section{CONCLUSIONES}

A continuación, enumeramos, a modo de conclusión, las implicaciones pedagógicas que podemos extraer del enfoque descrito en este trabajo:

1. Los alumnos aprenden a involucrarse activamente en el proceso de lectura.

2. Aprenden estrategias cognitivas y metacognitivas que les permitirán adquirir nuevos conocimientos, integrarlos en su sistema cognitivo y tener control sobre el proceso de aprendizaje.

3. Se acostumbran a adaptar las estrategias aprendidas a las diferentes situaciones y a hacerse sus propias preguntas de comprensión, todo lo cual contribuye a formar lectores/ alumnos más autónomos.

4. La discusión sobre las respuestas favorece la interacción entre alumnos y profesor, creando un clima relajado y al disminuir lo que Krashen llama "el filtro afectivo" se favorece el aprendizaje.

5. Este método estimula el sentido analítico y la intuición de nuestros alumnos, cualidades primordiales y complementarias en el proceso de aprendizaje.

6. Aprenden a centrar la atención en el significado del texto, dedicando menos esfuerzo a los aspectos lingüísticos vacíos de contenido; de esta forma leen más rápido, comprenden mejor y disfrutan leyendo en otro idioma.

7. Desarrolla su sentido crítico que les permitirá valorar las diferentes interpretaciones del texto, la intención del autor, la ironía, etc...

8. La adquisición del idioma resulta de una manera inconsciente, lo cual es más natural y divertido.

9. Aumenta la confianza del alumno en sus capacidades pues no sólo constata su progreso en el aprendizaje sino que se da cuenta de que puede usar y aplicar sus conocimientos a diferentes contextos. Como consecuencia de lo anterior los alumnos se sentirán más motivados ( Pintich, 1999; González, 1999).

10. Finalmente, el uso sistemático de las estrategias mencionadas permite al alumno aceptar su responsabilidad en el proceso de aprendizaje y le hace sentirse más implicado y motivado. El resultado es que el alumno relaciona la adquisición de conocimientos con el esfuerzo personal y con la aplicación de las estrategias aprendidas (Madrid,1999). De esta forma conseguimos formar alumnos motivados y preocupados por el proceso de aprendizaje.

\section{BIBLIOGRAFÍA}

Block, E. L. 1992. "See how they read: comprehension monitoring of L1 and L2 readers". TESOL Quarterly 26, 2: 319-342.

Bradawi, N. 1992. "The Reading Dilemma: Meeting Individual Needs". English Teaching Forum 30, 2: 6-35.

Casanave, Ch. P. 1988. "Comprehension monitoring in esl reading: a neglected essential”. TESOL Quarterly 22, 2: 283-301. 
Garner, R. 1988. Metacognition and Reading Comprehension. Norwood, N.J.: Ablex.

González, L. M. 1999. "Activities and techniques to motivate learners of English”.

Teaching and Research in English Language and Linguistics. Eds. P. Alonso, M. F.

García-Bermejo, M. J. Sánchez y C. Moran. León: Celarayn. 191-202.

Madrid, D. 1999. La Investigación de los Factores Motivacionales en el Aula de Idiomas. Granada: Grupo Editorial Universitario.

Nuttall, C. 1982. Teaching Reading Skills in a Foreign Language. London. Heinemann.

O’Malley, J. M. y A. U. Chamot. 1990. Learning Strategies in Second Language

Acquisition. New York: Cambridge University Press.

Oxford, R. 1990. Language Learning Strategies: What Every Teacher Should Know. New York: Newbury House/Harper \& Row.

Pintrich, P. R. 1999. "The role of motivation in promoting and sustaining self-regulated learning". International Journal of Educational Research 31, 6: 459-470.

Sorani, D. y A. R. Tamponi. 1992. "A cognitive approach to content-based instruction". English Teaching Forum 30, 1: 6-9.

Stevens, R. J. 1988. "Effects of strategy training on the identification of the main idea". Journal of Educational Psycology 80,1: 21-26.

Voss, J.F. y L. N. Silfies. 1996. "Learning from history text: the interaction of

knowledge and comprehension skill with text structure". Cogniton and Instruction 14, 1: 45-68.

Widdowson, H.G. 1978. Teaching Language as Communication. Oxford: OUP.

Yuill, N. y T. Joscelyne. 1988. "Effect of organizational clues and strategies on good and poor comprenhender's story understanding". Journal of Educational Psychology 80, 2: 152-158. 